\title{
Review Paper on Agent Oriented Traffic Coordination
}

\author{
Ankit Sharma \\ M-Tech Scholar, Department of \\ Computer and Science engineering, \\ Ajay Kumar Garg Engineering College, \\ Ghaziabad, India
}

\author{
Shashank Sahu \\ Associate Professor, Department of \\ Computer and Science Engineering \\ Ajay Kumar Garg Engineering College \\ , Ghaziabad, India
}

\begin{abstract}
Inclusion of Agents in the domain of managing traffic and its real time demand of coordination and cooperation is proven quite promising. Agent oriented traffic coordination helps in improving traffic management and in achieving efficiency of vehicle movement. Today with rapid urbanization and increasing number of vehicles traffic congestion has emerged out as a serious bottleneck. Automated vehicle decision models that are capable of taking self-decision finding a better path for the movement of traffic flow in control area are developed and more work is going on this field. These automated vehicles are able to communicate and coordinate with each other's thereby maintaining proper coordination among neighboring vehicles. Every neighbor knows its current location and its group member's.

This paper presents several traffic coordination, communication and controlling strategies and methods for controlling them. Use of agents in traffic simulation, helps in reducing traffic congestion and delay. Lack of communication and controlling mechanism decreases traffic speed and average target destination time.
\end{abstract}

\section{Keywords}

Agent coordination, vehicle grouping, MAS cooperation

\section{INTRODUCTION}

Agent Software system is a large body of autonomous agents, which performs necessary action itself without human intervention. An agent is self-functioning, independent and always functional. It is a software program that performs required computation and tasks on behalf of the user without involving them. Autonomy, collaboration and reactivity are the most appealing characteristics of agent these properties help in development of automated traffic managing systems [1].

Role of agents in real time applications and monitoring day to day scenarios helps us to analyze and find out suitable solutions. Communication among vehicles will give them the ability to organize traffic and make decision autonomously. Urban traffic is a non-linear, vague, energizing and convoluted system. Traffic control system is a distributed system and its subsystem needs to be cooperative in order to achieve coordination control for traffic flow in specified area.

The ongoing work on multi-agent technology significantly enhances the designing and characterization of problem domain these are:

\section{Geographically distributed domain.}

2. Dynamic environment existing in sub-systems.

Multi-agent technology has higher capability of problem solving than a single Agent. Centralized approach in traffic management does not proven success in managing traffic. Because centralizing a system need specific areas for deployment of coordinating agent that increases the cost also.Today traffic problem is affecting many countries and the approach to manage them does not lie in the use of sensory product. As with rapid advancement in technology vehicles are capable of being coordinated and communicated. For agents to be properly coordinated there should be a communication among agents. These autonomous vehicle agents pass message among neighboring vehicles with proper id and message.

\section{LITERATURE SURVEY}

Anshul Verma et al. 2015 discussed As with growing complexities in managing traffic in every domain of real world either in road traffic management, train based management, or in air traffic controlling use of multi-agent technology seems quite interesting. They pointed out the measurement that should be applied to the safety of railway system [2]. The behavioral analysis need to be taken into consideration when designing system for train safety, the scene that came into light when train starts running, will be helpful in summarizing the response of the system to these conditions.

RFID tag is also helpful for managing and sensing the movement of autonomous vehicles they are being traced and their coordination and cooperation patterns can be used for the behavioral analysis [3].A study through simulation helps in determining the behavior of the traffic model to achieve moving goal. The work done here is to achieve the objectives that there should be no collision between moving authorities and to analyze the proactive nature of IRMS [4].

The static specification that are deterministic, the system has prior learning for that, but in case of dynamic parameters they are pointed out and analyzed at runtime this is a challenging task to estimate moving authority .For analyzing the behavioral analysis of the traffic model multiple scenarios have been modeled. The practical analysis is done on the data collected over actual fault data that help in properly estimating the behavior of traffic participants in the traffic model.

Jana Gormer et al.2013 have given their concern about the group-oriented traffic coordination and their approach of forming group in a distributed traffic environment. Group formation of vehicle agents to coordinate either lane on driving or their speed, needs to be evaluated. For smoother traffic movement, giving higher speeds and less time to reach to the target destination, a decentralized approach relying on cooperative driving strategy will prove a favorable approach[5],[6].

Vehicle agent that are developed with group oriented driving method are capable of perceiving their environment and exchange information often when traffic participants move according to its preferences their movement is restricted by others. Problems of lane switching and fast vehicles are being forced to move slowly because of slow vehicles. The 
approach described in this is GOD which allows cooperative driving between autonomous vehicle agents. They have the ability to perceive their nearby area, can communicate and can form group to avoid conflict situations [7].

\section{DECENTRALIZED VEHICLEG ROUPING}

The main advantage of using decentralized approach is it decreases the overhead of installing extra devices; there is no need of central controller. The centralized approach is limited in communication and computation; it is not possible for a central coordinator to coordinate with all vehicles. The dissimilarity function is one of the criteria of group formation that allow other neighboring members to be included in the group if they satisfy the range of dissimilarity function. Vehicle group $\mathrm{G}_{\mathrm{x}}$ is defined with leader $\mathrm{x}\left(\mathrm{id}_{\mathrm{x}} ; \mathrm{a}_{\mathrm{x}} ; \mathrm{d}_{\mathrm{x}} ; \mathrm{d}_{\mathrm{sx}}\right)$ as set containing vehicles $y$ with following property:

$$
\forall_{\mathrm{y}} \in \mathrm{G}_{\mathrm{x}}, \mathrm{f}(\mathrm{x}, \mathrm{y})<=\alpha_{\mathrm{x}}
$$

$\alpha_{x}$ is a fixed value predefined by $x$. The attributes $i_{x} ; a_{x} ; d_{x}$; $\mathrm{d}_{\mathrm{sx}}$ are identifier number, maximal acceleration rate, maximal deceleration rate, and desired speed of $\mathrm{x}$. Function $\mathrm{f}(\mathrm{x} ; \mathrm{y})$ used to calculate the dissimilarity between vehicles $\mathrm{x}$ and $\mathrm{y}$ in terms of their maximal deceleration rate, maximal acceleration rate, and desired speed. Thus, a group leader $\mathrm{x}$ will accept any vehicle $\mathrm{y}$ as its member if the dissimilarity $\mathrm{f}(\mathrm{x}$; $\mathrm{y}$ ) is smaller than or equals $\alpha_{\mathrm{x}}$ Using static attributes as inputs for the dissimilarity function $\mathrm{f}(\mathrm{x} ; \mathrm{y})$, a group leader $\mathrm{x}$ can decide if a vehicle $y$ can participate in its group or not.

\section{GROUP CONFLICTS AND SOLUTION}

There might be a situation when group conflicts occur, while group formation that needs to be taken care. The conflict is detected by leader of group. For this the leader or member maintain information about its own group. For finding groups in conflict a group member send information to its neighboring group. By getting the response from neighbor a binary function is calculated.

$$
\begin{aligned}
& \text { Conf }\left(G_{\mathrm{x}}, \mathrm{G}_{\mathrm{z}}\right)=\sqrt{\text { yes }} \quad \text { if } \mathrm{ds}_{\mathrm{x}}-\mathrm{ds}_{\mathrm{z}}>\mathrm{wd}_{\mathrm{s}, \mathrm{x}} \wedge \\
& \begin{array}{ll}
\exists z_{n} \in G_{z}, P & P \\
\text { No } & \text { otherwise. }
\end{array}
\end{aligned}
$$

where $\mathrm{Px}_{\mathrm{a}}$ and $\mathrm{Pz}_{\mathrm{n}}$ are positions of $\mathrm{x}_{\mathrm{a}}$ and $\mathrm{z}_{\mathrm{n}}$ on the highway. Once a collision is detected $\left(\operatorname{conf}\left(\mathrm{G}_{\mathrm{x}} ; \mathrm{G}_{\mathrm{z}}\right)\right), \mathrm{x}_{\mathrm{a}}$ sends information of group $G_{z}$ to the leader of group that was also involved in the conflict. Leader regulates its member via group lane. Group lanes are specifically pre-occupied for vehicle group's members. The group lane choice is based on following two criteria:

1) Lane changing of members should be as few as possible. 2) The group lanes should warranty that fast groups are not blocked by slow groups. The paper lacks in pointing out study of application of group life and whether there should be one group or multiple groups, and also coordination architectures and strategies.

Khaled Almejalli et al. 2009 in this paper they presented that there is a strong interrelation among traffic scenarios at multiple areas of a road network. If one tries to solve a particular area problem, this might create chaos at another location, even though we have applied traffic management strategies, the situation may worse or remain the same.

Therefore, coordination among traffic participants is needed to fulfill the same goal. This paper has proposed an intelligent decision support system relying on multi-agent so that it can provide support to the traffic control center of road for managing the ongoing traffic participants [8] The road traffic network is sub-divided into sub-networks providing every network having its evaluation Agent There is a high level agent, that work as coordinator, that control all the traffic movement and helps in finding optimal solution to traffic control using intelligent agent [9],[10].The architecture used for traffic control system is centralized coordinator. That keeps in mind the interrelation between the traffic action and controlling them.

Feng chen et al. 2008 according to him:-Urban traffic having high uncertainty, non-linearity in nature and puzzled system. The main aim of traffic control for urban road network is to achieve wide range of coordination for the existing network. Multi-agent system automation is considered as an efficient approach for distributed control. For controlling urban traffic and its coordination, MAS control mechanism is to be used. Cooperation between vehicles on the network communication requirements need to be met out among heterogeneous agent.

Traditional approach of handling increasing traffic demand is difficult to implement now a day excellent controlling of traffic flow is achieved through mathematical modeling intelligent control technology also applied in controlling traffic system, particularly in the field of area control. System use for managing traffic is a distributed system and also its subsequent system has to be cooperative in achieving area coordination of traffic movement [11], [12]. Masond had put the proposal of using multi-agent system developed signal control at intersection [13].The agent architecture and communication aspects are not taken into consideration. Cooperative control mechanism needs to be developed for managing distributed traffic system and traffic control. The coordination of agent study is based on Q- learning but the disadvantage of this approach is that it includes high computation [14].In this paper the task of area coordination among agent is divided in to local intersection control task, the task of coordination is handled by area control agent and intersection agent. The signal cycle is optimized by area control agent for intersection agent and furthermore signal timing has been performed by intersection agent in respective of dynamic traffic ratio. This coordination can decrease the queue of traffic and average delay of traffic movement.

R.T. van Katwijk et al. 2005, presented problem related to road traffic management by presenting a test bed for multiagent control systems. The increase in number of traffic on a network makes it difficult for a large number of traffic management instruments to coordinate. An approach to handle this complexity is by dividing this coordination into successive problems. Multi-agents help in distributing the problem and facilitate the coordination activities of the agents when needed. Any consensus about any configuration for managing traffic through multi-agent system and the manner in which the activities of the agent that includes multi-agent system needs to be properly coordinated.

To overcome from the problem of road traffic management for multi-agent control a software system needed that will handle all controlling mechanism [15].

The agent based traffic management can be presented as a test bed which has greater influence over road traffic. FIPA standard has been used for traffic management implemented using test bed for multi-agent control system. The FIPA standards help in configuration of multi-agent system [16] The transformation of traffic managed using multi-agent 
system to real application can be done using FIPA standards compliance

The presented test bed approach enables the development of tool for studying the applicability in traffic management of multi-agent systems. This paper has given coordination control approach for traffic management and the simulation result calculated shows that the randomness in the vehicle arrival process from multiple directions will not give a desired pattern at the intersection point of vehicles, according to R.T. Van Katwijk et al. Future work will be focused from network to grid network.

\section{TABLE OF COMPARISON}

\begin{tabular}{|l|l|}
\hline PAPER & PROPOSED WORK \\
\hline AnshulVerma et al. 2015 & $\begin{array}{l}\text { Safety issue is addressed and } \\
\text { the behavior analysis is also } \\
\text { considered for train safety. }\end{array}$ \\
\hline Jana Gormer et al. 2013 & $\begin{array}{l}\text { Focused on the movement of } \\
\text { vehicle by group formation } \\
\text { and mathematical approach is } \\
\text { used. }\end{array}$ \\
\hline Khaled Almejalli et. al2009 & $\begin{array}{l}\text { The network is sub-divided; } \\
\text { each having its own } \\
\text { evaluation agent, intelligent } \\
\text { decision support system is } \\
\text { used. }\end{array}$ \\
\hline Feng chen et al. 2008 & $\begin{array}{l}\text { The area coordination control } \\
\text { task handled by control agent } \\
\text { and intersection agent by } \\
\text { using fuzzy concept. }\end{array}$ \\
\hline R.T. van Katwijk et al. 2005 & $\begin{array}{l}\text { The FIPA standard has been } \\
\text { used which helps in traffic } \\
\text { coordination implemented } \\
\text { using test bed for multi-agent } \\
\text { control system. }\end{array}$ \\
\hline
\end{tabular}

\section{CONCLUSION}

Formation of groups of vehicles moving on a road in a highly crowded area will be helpful in achieving efficiency of traffic movement. Group oriented driving allows each member of its group and neighboring groups to be properly coordinated by knowing all its member location. Leader takes the responsibility of group formation and resolving the group conflicts .several researchers have proposed the coordination and communication methods and structures, their research work mainly focused on this but they failed in pointing out exact solution of real time traffic scenarios reference of papers. Each car on a road must follow a set of rules if their slower car in front of them they must slow down their speed and if no car they should increase their speed. The movement of a car in a group is much different than an individual car that makes up a group. There are certain things to try and that should be taken in to account as if we give different rules to the acceleration /deceleration values to some of the cars, what is the effect of these changes on the formation of traffic jam, group life time needs to be kept in to account as when the group formation takes place and till what time. The lanechanging behaviors are the most challenging that were to be addressed.

Resulting vehicle behavior is then mostly realistic especially at the macroscopic level; resulting behavior of vehicle is more realistic however, that can be improved by further analysis. The characteristics of Driver not modelled and they are expected to keep their safety and follows proposed driving model; there are two types of models exists real world, one is the user- driven and other is vehicle - driven scenario. In this vehicles act as intelligent agents in both of them.

\section{REFERENCES}

[1] F. Y. Wang, "Toward a revolution in transportation operations: AI for complex systems," IEEE Intell. Syst.,vol. 23, no. 6, pp. 813,Nov./Dec.2008

[2] Multi-agent communication-based train control system for Indian railways: the behavioral analysis Anshul Verma1• K. K. Pattanaik1 February 2015.

[3] EURESCOM Analysis of existing agent-oriented tools,(2001).

[4] Khan MS, Benkrid K (2010) Development techniques of multiagents based autonomous railway vehicles control systems. Word AcadSciEng Technol.

[5] A. L. C. Bazzan, D. de Oliveira, and B. C. da Silva, "Learning in groups of traffic signals," Eng. Appl. Artif. Intell., 2010.

[6] H. Prothmann, J. Branke, H. Schmeck, S. Tomforde, F. Rochner, J. H"ahner, and C. M"uller-Schloer, "Organic traffic light control for urban road networks,'International Journal of Autonomous and Adaptive Communications Systems, 2009.

[7] A. Lansdowne, "Traffic simulation using agent-based modelling," Science, 2006.

[8] K. Almejalli, K. Dahal, and A. Hossain, "Intelligent Traffic Control Decision Support System," Lecture Notesin Computer Science, 2007.

[9] B. De Schutter, S. P. Hoogendoorn, H. Schuurman, and S. Stramigioli,"A Multi-Agent Case-Based Traffic Control Scenario Evaluation System," in IEEE IntelligentTransportation Systems.vol. 1, Schangai, 2003.

[10] F. Logi and S. G. Ritchie, "A Multi-Agent Architecture for Cooperative Inter-Jurisdictional Traffic Congestion Management," Transportation Research Pa C: Emerging Technologies, 2002.

[11] Asim Karim, "CBR Model for Highway Work Zone Traffic Management," Journal of TransportationEngineering, 2003.

[12] HaitaoOu; Weidong Zhang; Wenjing Zhang; Xiaoming $\mathrm{Xu}$, "Urban traffic multi-agent system based on RMM and Bayesian learning," Proceedings of American Control Conference, June 2000.

[13] Mohammadian M., "Multi-Agents Systems for Intelligent Control of Traffic Signals, Computational Intelligence for Modeling," Control and Automation, 2006 International Conference on Intelligent Agents, Web Technologies and Internet Commerce, Nov. 2006.

[14] Zhang Hui, Chen Yangdan, Yang Yuzhen, et al, "Urban Traffic Coordination Control System Based on MultiAgent," Computer and Communications, 2006.

[15] J.Cuena, J. Hern'andez, and M. Molina. Knowledge October 1995

[16] FIPA. The foundation for intelligent physical agents.2003. 